\title{
REGENERATIVE POTENTIAL OF SIMVASTATIN ON DENTAL PULP STEM CELLS IN DOG'S TEETH: A HISTOLOGIC AND RADIOGRAPHIC STUDY
}

\author{
Bayuomi A. Amr", Islam M. Taher**, Al-Khawas A. Moataz ${ }^{* * *}$, Gobran G. Hany****
}

\begin{abstract}
Objective: This study was directed to evaluate the regenerative potential of simvastatin (SIM) on dental pulp stem cells (DPSCs) in non-vital immature dogs' teeth both histologically and radiographically. Methods: The present study was carried out on 6 adult healthy male mongrel dogs aged from 4 to 6 months and their weight ranged from 12 to $17 \mathrm{~kg}$. Three premolars (two rooted) in each quadrant were included in this study summing up the total number of teeth to 72 (12 premolars x 6 dogs). Endodontic access cavities were prepared in all experimental teeth in the buccal surface and the vital pulps of teeth were exposed till confirmation of radiolucency. Then triple antibiotic paste was used for disinfection of root canals for 3 weeks. After disinfection period, the teeth were divided into two groups: group 1; treated with dental pulp stem cells and simvastatin while group 2; was treated with dental pulp stem cells only. Both groups were evaluated histologically and radiographically after 1 week, 1 month and 3 months. Results: Better radiographic and histologic findings were found in the group treated with dental pulp stem cells with simvastatin than groups treated with dental pulp stem cells only after 1 week, 1 month and 3 months of evaluation periods. Conclusions: Under the condition of this study, simvastatin aids in regeneration of infected immature dog's teeth with the ability of producing functional dentin-pulp complex and inclusion of a stem cells seemed to improve the outcomes of attempted regeneration.
\end{abstract}

Key words: Simvastatin, Dental pulp stem cells, Immature dog's teeth, Regenerative Endodontics

\section{INTRODUCTION}

Treatment of immature teeth with necrotic pulps has been considered a big challenge because interruption in root development leads to weak and thin fragile dentinal walls liable to fracture, beside the difficulty to achieve an adequate apical seal using conventional root canal filling techniques. Traditional management of such teeth ranges from induction of apical closure using calcium hydroxide (Apexification), apical plug using bioactive materials such as MTA, to apical surgery with retrograde sealing ${ }^{(1)}$. Recently, regenerative endodontics has gained attention as a biologically based alternative as it can allow for further root maturation in length and thickness by the regenerated vital tissue. To complete this procedure successfully, three components including stem cells, scaffolds and growth factors should be involved in such protocol ${ }^{(2)}$.

Recently, Simvastatin (SIM), a widely accepted drug used primarily to lower the cholesterol levels has revealed increase in bone mineral density with long term systemic administration in humans (3). Furthermore, simvastatin-treated dental pulp stem cells exhibited enhanced odontogenic differentiation and accelerated mineralized tissue formation (4). This observation led to the possibility of utilizing Dental Pulp Stem Cells (DPSCs) in combination

\footnotetext{
*Assistant Lecturer of Endodontics, Faculty of Dentistry, Al-Azhar University

** Professor of Endodontics, Faculty of Dentistry, Al-Azhar University

*** Assistant Professor of Endodontics, Faculty of Dentistry, Al-Azhar University

***** Assistant Professor of Oral biology, Faculty of Dentistry, Al-Azhar University
} 
with simvastatin to regenerate pulp and dentin for clinical applications ${ }^{(5)}$. From the histological point of view, the nature of the tissue formed in the canal space in human revascularized immature permanent teeth with apical periodontitis is speculative because no histologic studies are available ${ }^{(6)}$. Therefore, animal models may shed some light to address this issue. The combination of simvastatin with stem cells from different sources to restore a functional dentin-pulp complex is not fully investigated in dental literature.

\section{MATERIALS AND METHODS}

The procedures were carried out in the Department of Veterinary Surgery, General veterinary hospital in Alabbasyya, Cairo, Egypt. Six adult healthy male mongrel dogs aged from 4 to 6 months and their weight ranged from 12 to $17 \mathrm{~kg}$ were used in this study. The dogs were examined by the staff member of Veterinary Surgery to rule out the presence of any disease. Dogs were vaccinated by the triple vaccine against rabies, distemper, leptospirosis and hepatitic viruses.

Three premolars (two rooted) in each quadrant were included in this study summing up the total number of teeth to 72 (12 premolars x 6 dogs). Under dog's sedation, clinical and radiographic examination was done to confirm shedding of the primary teeth with eruption of their permanent successors with incomplete root formation. All the dogs were pre-medicated with subcutaneous injection of atropine sulphate $0.05 \mathrm{mg} / \mathrm{kg}$ body weight (Atropine Sulphate; Misr Co., Cairo, Egypt) and intramuscular Xylazine $\mathrm{HCl} 1.1 \mathrm{mg} / \mathrm{kg}$ body weight (Xylaject; ADWIA Co., Cairo, Egypt), Diazepam $0.5 \mathrm{mg} / \mathrm{kg}$ (Diazepam Bayer, Leverkusen, Germany) and Amoxicillin and flucsacillin (Flumox $1000 \mathrm{mg}$ vial E.I.P.I.Co 10th of Ramadan Egypt) as a prophylactic antibiotic. Then the anesthesia was induced by intravenous Ketamine $\mathrm{HCl}$ $5 \mathrm{mg} / 1 \mathrm{~kg}$ body weight (EIMC. Pharmaceuticals co., Egypt) and maintained by $25 \mathrm{mg} / 1 \mathrm{~kg}$ intravenous incremental doses of $2.5 \%$ solution of Thiopental sodium (EIPICO, Cairo, Egypt) until loss of pedal and corneal reflexes, constriction of the pupils and development of shallow regular respiration.

In the first visit, endodontic access cavities were prepared in all experimental teeth in the buccal surface and the vital pulps of teeth were exposed. This procedure was performed with high-speed hand piece (NSK, Tokyo, Japan) under constant coolant with a size no. 2 sterile carbide bur (Brassler USA, Savannah, Georgia). Then a size 25 sterile k-file (Mani, Inc., Tochigi, Japan) was used to disrupt the pulp tissue in the canals followed by placement of a piece of cotton into the entrance of each canal. In the second visit, rubber dam isolation of the selected teeth was done and root canals were irrigated using $10 \mathrm{~mL}$ of $1.5 \%$ sodium hypochlorite (Regular household Bleach, Clorox, Egypt), flushed with $10 \mathrm{ml}$ of sterile saline $(0.9 \%$ sodium chloride, Novartis, Egypt) and dried with sterile paper points. The triple antibiotic paste (TAP) was prepared using metronidazole $500 \mathrm{mg}$ tablets (Flagyl 500 $\mathrm{mg}$, Aventis, Cairo, Egypt), ciprofloxacin $250 \mathrm{mg}$ tablets (Ciprocin $250 \mathrm{mg}$, EPICO, Cairo, Egypt) and doxycycline $100 \mathrm{mg}$ capsules (Vibramycin, Pfizer, Cairo, Egypt) in equal portions of each antibiotic by concentration mixed with 3-4 drops of sterile saline to a paste like consistency. TAP was injected into the canal using a 16-gauge needle and the access cavities were sealed with a resin reinforced glass ionomer. After disinfection period (3 weeks), antibiotic paste was removed by copious irrigation using $10 \mathrm{~mL}$ of $1.5 \%$ sodium hypochlorite then flushed with normal saline using a 27 -gauge side vented closed end endodontic irrigating needle. A $17 \%$ EDTA liquid was used as final rinse then the root canals were dried using paper points.

According to sample size calculation, the number of selected teeth included within the study was set to be 72 teeth included in 6 dogs. Teeth were divided into four equal groups according to different treatment protocols used for each experimental group as follows: 
Group 1: treated with dental Pulp Stem Cells (DPSCs) and simvastatin (SIM) hydrogel (36 teeth)

Group 2: treated with Dental Pulp Stem Cells (DPSCs) (36 teeth)

Then each main group was subdivided into 3 subgroups according to post treatment evaluation periods as follows:

Group 1: evaluation period after 1 week of treatment (24 teeth)

Group 2: evaluation period after 1 month of treatment (24 teeth)

Group 3: evaluation period after 3 months of treatment (24 teeth)

\section{Tissue harvesting and isolation of dental pulp stem cells:}

Extraction of maxillary lateral incisor with vital pulp was done from each dog to isolate dental pulp stem cells. A longitudinal inciso-apical groove and vertical split was done then the pulp was picked up using toothed forceps. The harvested pulp was placed in a minimum essential medium (MEM) transporting media (Sigma Aldrich, St. Louis, MO, USA) in a validated, temperature-controlled $2^{\circ} \mathrm{C}$ transport box fitted with a frozen cold pack and shipped to the stem cells laboratory for processing. The harvested dental pulp was removed from the transport media, washed in Phosphate buffered Saline (PBS) to ensure removal of any exogenous debris, placed in a sterile petri dishes and cut into small pieces using a sterile scalpel blade. Enzymatic digestion of tissue was done as described by Gronthos et al. ${ }^{(14)}$ where $3 \mathrm{mg} / \mathrm{ml}$ collagenase type I were used as digesting solution. The harvested tissues were then incubated at $37^{\circ} \mathrm{C}$ in a humidified atmosphere containing $5 \%$ $\mathrm{CO}_{2}$ for the time necessary to allow cells to slip down from the explants. After incubation, the digestive reaction was stopped by the addition of fresh basic medium which was added to neutralize collagenase. The tubes were then centrifuged for five minutes at room temperature to concentrate cells at the bottom of the tubes. A sterile serological pipette was used to transfer the medium containing cells into T-25 cell culture flask (Falcon, Franklin Lakes, NJ) with filter top cap. The flask was then incubated at $37^{\circ} \mathrm{C}$ in a humidified atmosphere containing $5 \% \mathrm{CO}_{2}$ for the time necessary for adherence. After washing the cells with Hanks Balanced Salt Solution (HBSS), $1 \mathrm{ml}$ of Trypsin was added to the flasks in noncell side to detach the cells from the flask floor (trypsinization). Then $5 \mathrm{ml}$ of medium was added to the flask and trypsin/medium/cell suspension had been collected in $15 \mathrm{ml}$ test tube for centrifuging at $600 \mathrm{rpm}$ for 6 minutes. Suspension was vacuumed out to get out as close as possible to the pellet without touching the cells. Then splitting of the cell suspension was done equally in T 25 flasks and the flasks were put on their sides in the incubator with loose caps. After 7 days, the cells were observed under microscope and non-adherent cells were discarded. In these experimental conditions the cells reached confluence within 15-20 days of culturing through three passages. Counting of the cells was done using hemocytometer and Trypan blue stain was used to detect viable cell count. With the aid of inverted phase contrast light microscope, all the viable (big, rounded) cells were counted from the cell suspension.

\section{Preparation of Simvastatin hydrogel}

Simvastatin was dissolved in absolute ethanol (50 mg: $1 \mathrm{ml}$ ) and mixed with a magnetic stirrer then stored at $4^{\circ} \mathrm{C}$ for future use. The selected dose was $0.1 \mu \mathrm{l} / \mathrm{L}$ of simvastatin. Methylcellulose gel (MC) (Guangzhou Qiyun Biotechnology, China) served as the simvastatin delivery system in which $4.0 \%(\mathrm{w} / \mathrm{v})$ methylcellulose was dissolved in hot triple-distilled water to produce methylcellulose gel, which was used as the simvastatin carrier after it cooled down. DPSCs were transplanted as previously described by Krebsbach et al., $1997^{(7)}$ and exposed to $0.1 \mu \mathrm{ml} / \mathrm{L}$ simvastatin hydrogel ${ }^{(8)}$. 


\section{Euthanasia of the dogs:}

After each evaluation period, the dogs were sacrificed under general anesthesia provided by pentobarbital where The carotid arteries were exposed and cannulated then the dogs were euthanized with additional pentobarbital at a dose of $90 \mathrm{mg} / \mathrm{kg}$ IV. The animals were perfused with $10 \%$ buffered formalin (Fisher Scientific, Fair Lawn, New Jersey).

\section{Histologic preparation and evaluation:}

After euthanasia of the dogs, the maxillary and mandibular jaws were surgically removed and divided at the midline into two halves right and left one using a bone saw. The jaws with the involved teeth were decalcified, dehydrated and sections were made longitudinally every $5 \mu \mathrm{m}$ through the apical foramen of the roots. Tissues were stained with Hematoxylin and Eosin (H\&E) and evaluated under light microscopy at up to $400 \mathrm{X}$ magnification for the presence or absence of the newly formed tissues. Each individual root was taken as a unit of measurement/assessment and graded for the inflammatory changes, intracanal tissue changes, hard tissue formation and apical closure.

Statistical analysis was performed with IBM ${ }^{\circledR}$ SPSS ${ }^{\circledR}$ Statistics Version (Statistical Packages for the Social Sciences 20, IBM, Armonk, NY, USA). Data were represented by total number and percentage for each method used in the study. Descriptive statistics was done using one way ANOVA and Pearson correlation (r) tests for comparing the relation between different groups. Significance was set at $\mathrm{P}$ value $\leq 0.05$.

\section{RESULTS}

\section{Evaluation of the histological findings:}

Histological comparison among the tested groups after different evaluation periods

\section{- Inflammatory changes}

For both groups, there was a statistically significant inflammatory changes between different periods one weak, one month and 3 months. For one weak, one month and 3 months, there was no statistically significant difference between the two groups.

\section{- Intracanal tissue changes}

For both groups, there was no statistically significant changes between different periods one weak, one month and 3 months. While for one weak, one month and 3 months, there was a statistically significant difference between both groups. DPSCs+SIM recorded the better change.

\section{- Hard tissue changes}

For both groups, there was a statistically significant changes between different periods at one weak, one month and 3 months. There was no any difference between one month and 3 months. For one month and 3 months, there was a statistically significant difference between the both groups. For 3 months, there was no a statistically significant difference between (DPSCS+SIM, DPSCS).

\section{- Apical closure}

For both groups there was a statistically significant difference between one weak, month and three months as better apical closure was recorded at 3 months followed by one month. 


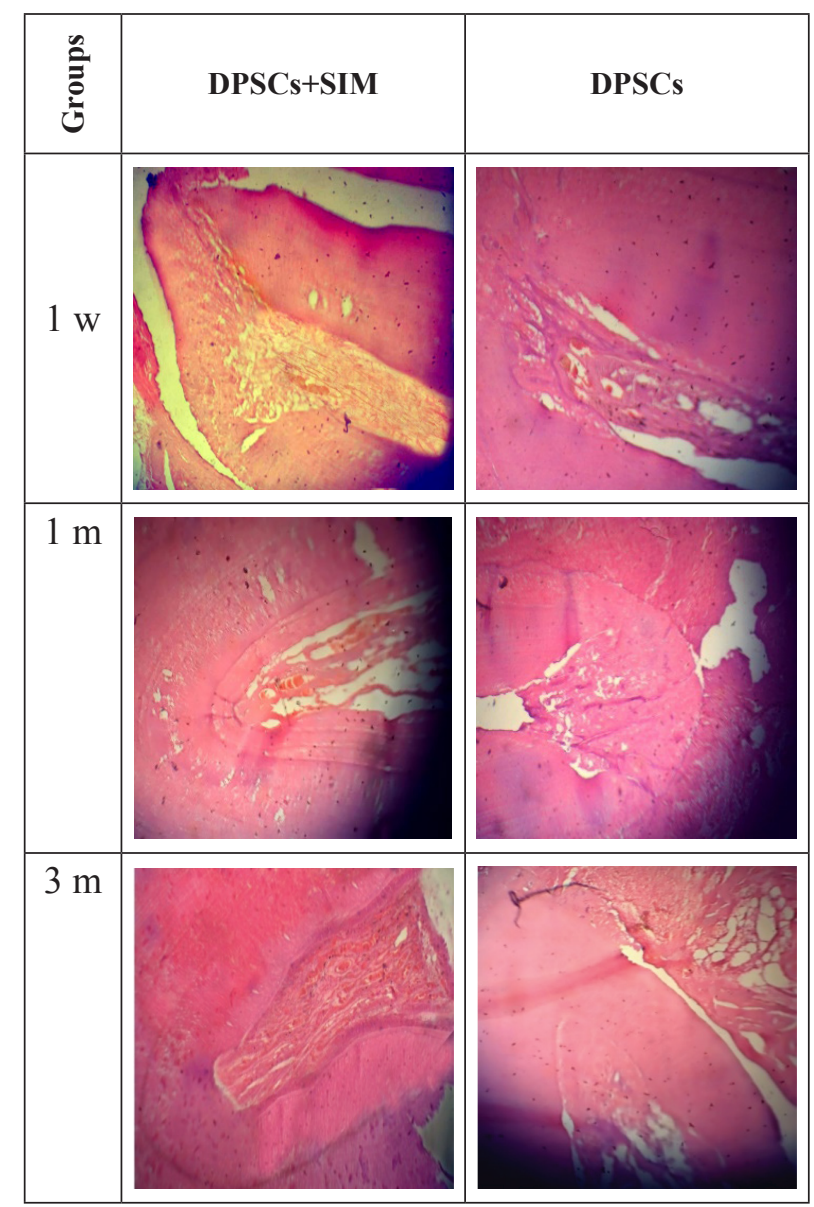

FIG (1): Histological sections of the groups over different evaluation periods:

\section{Evaluation of the radiographic findings:}

\section{Radiographic comparison among the tested groups after different evaluation periods}

\section{- Radiolucency:}

For DPSCs+SIM, there was a statistically significant difference between one week, one month and three months as better radiolucency decrease was recorded at 3 months followed by one month. For DPSCs, there was no statistically significant difference between one week, one month and three months. For one week, there was no statistically significant difference between both groups. For one month, there was no statistically significant difference between both groups. DPSCs+SIM showed the better radiolucency decrease after 3 months.

\section{- Apical closure}

For DPSCs + SIM, there was a statistically significant difference between one week, one month and three months. Better apical closure was recorded at 3 months and one month followed by one week. For DPSCs, there was no statistically significant difference between one week, one month and three months. For 3 months, there was a statistically significant difference between both groups. DPSCs+SIM showed the better radiolucency decrease followed by DPSCs.

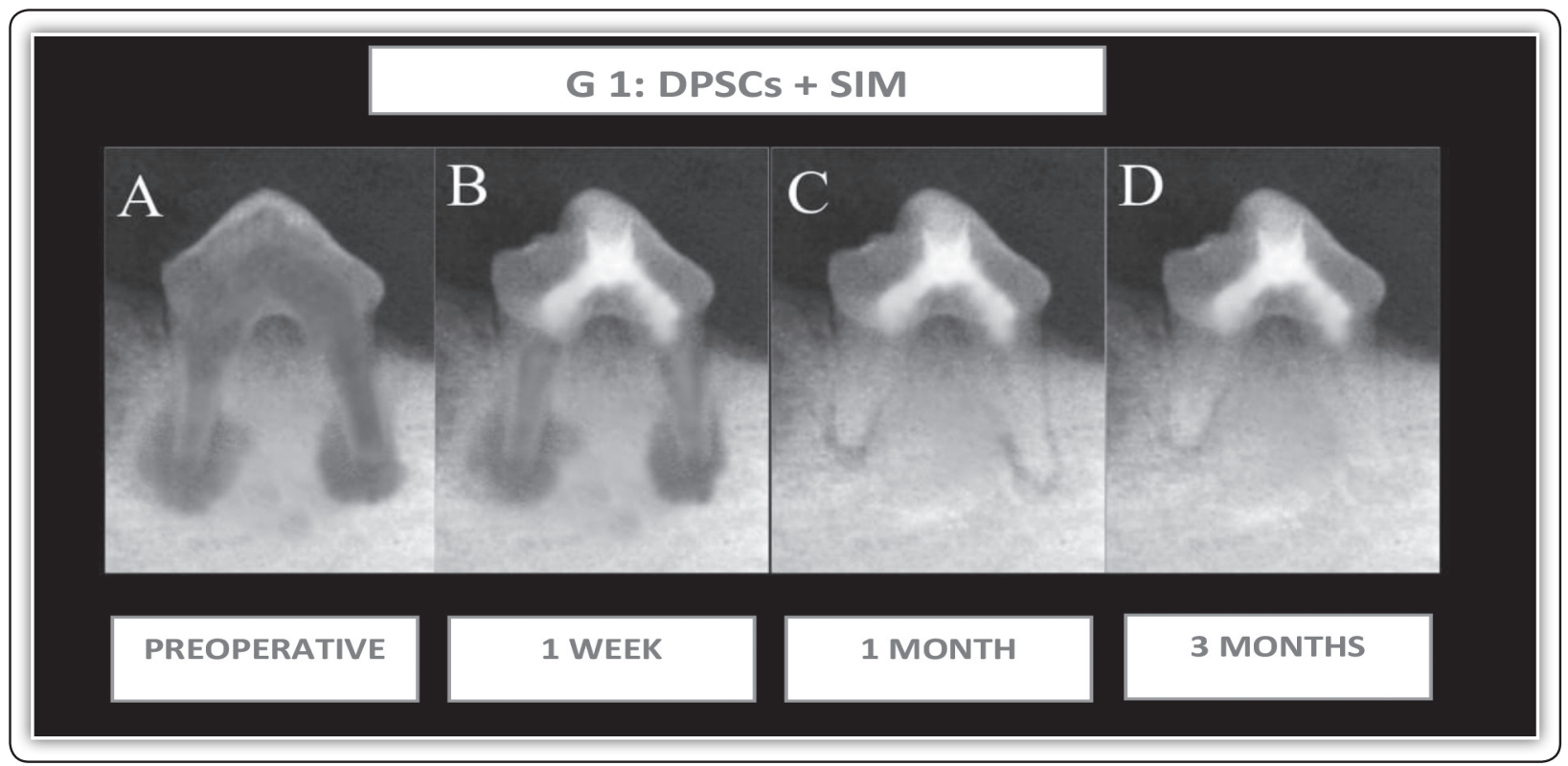

FIG (2) Group 1: Teeth treated with dental pulp stem cells added to simvastatin. Radiographic changes of group 1 over different evaluation periods 


\section{- Canal calcification}

For DPSCs+SIM, there was a statistically significant difference between one week, one month and three months as. canal calcification was significant at 3 months and one month when compared with one week. For DPSCs, there was no statistically significant difference between one week, one month and three months.

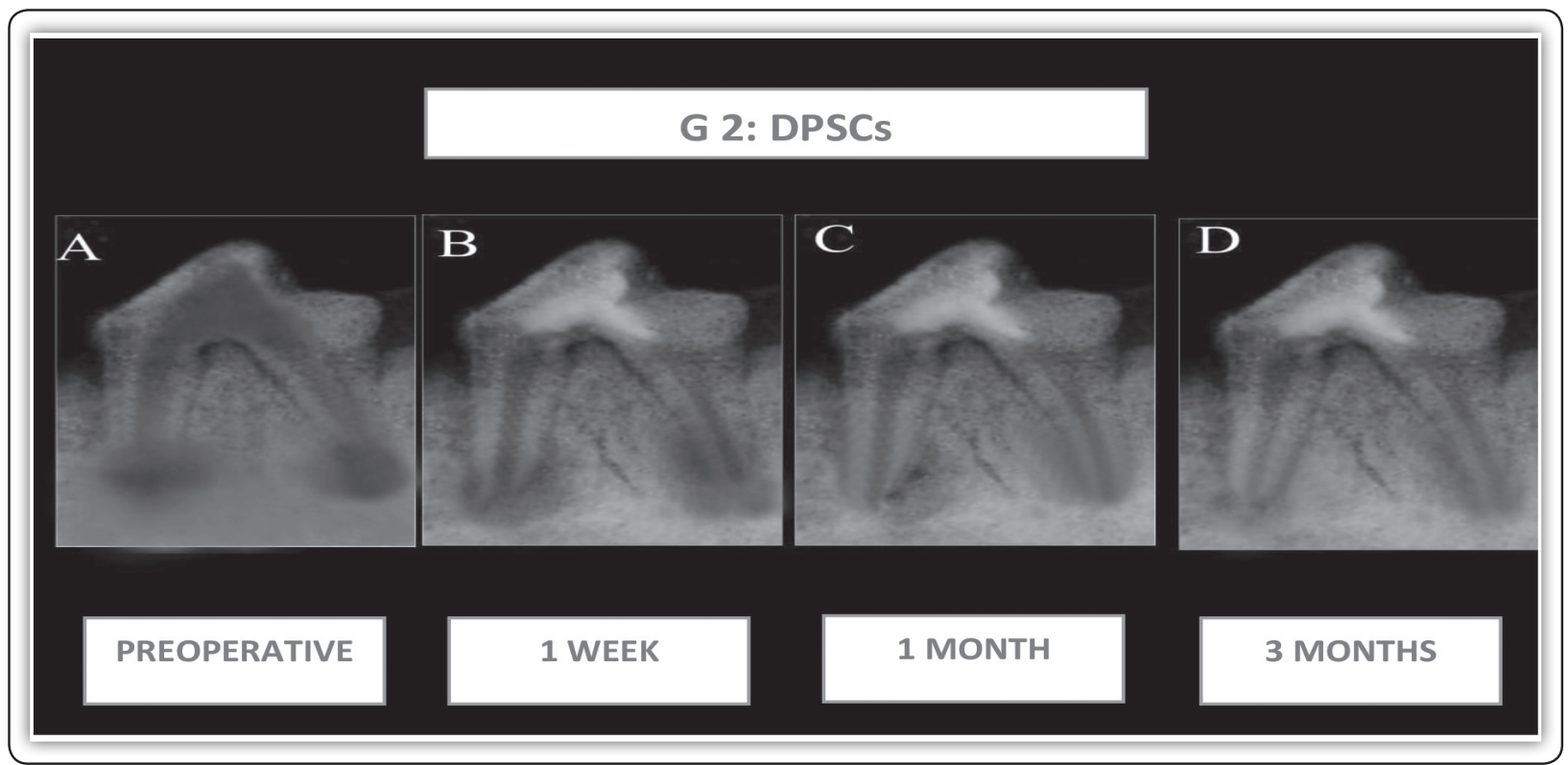

FIG (3) Group 2: Teeth treated with dental pulp stem cells. Radiographic changes of group 3 over different evaluation periods

\section{DISCUSSION}

Treatment of immature teeth with necrotic pulps represents a big challenge because interruption in root development leads to weak and thin fragile dentinal walls with difficulty to achieve an adequate apical seal using conventional techniques. Recently, regenerative endodontics has gained attention as a biologically based alternative as it can allow for further root maturation by the regenerated vital tissue ${ }^{(9)}$. The present study is an experimental study done to evaluate the treatment outcomes of immature teeth with necrotic pulps of dogs using injectable scaffold of simvastatin in combination with dental pulp stem cells or adipose-derived stem cells. Both radiographic and histologic evaluations were used to overcome the limitations of each method.

The choice of dogs as an animal model for biological experiments in endodontics is based on the fact that they have similar apical repair compared with humans but over a short duration (average one-sixth of human) due to the high growth rate. Also, dogs have a close similarity in radicular structure to immature human teeth in their open apex characteristics ${ }^{(10)}$. Two rooted teeth were selected in order to their accessibility for endodontic procedures and they have average-sized canals for endodontic manipulation. The age of the dogs ranged between 4 and 6 months which was suitable for the study of immature teeth, as premolar teeth are immature at this age range and the animals can withstand general anesthesia and surgical procedures. With regards to root canal disinfection, a concentration of $1.5 \%$ of NaOCL was used as it has the least cytotoxic effect on the stem cells compared to other concentrations ${ }^{(11)}$. The disinfection period was 3 weeks which was recommended by most of the published articles ${ }^{(12)}$. Final irrigation was 
done by $17 \%$ EDTA in order to expose growth factors entrapped in the dentin matrix including, bone morphogenic protein 2 (BMP2), Transferring Growth Factor-B (TGF-B) and angiogenic factors as platelets-derived growth factor (PDGF), vascular endothelial growth factor (VEGF) and fibroblast growth factor 2 (FGF 2$)^{(13)}$. It has been shown that EDTA-soluble factors stimulate matrix secretion, odontoblast differentiation, and tertiary dentin formation ${ }^{(14)}$. Many authors advocated non instrumentation technique as instrumentation could not only increase fragility of dentin walls but also injure stem cells present in the apical area of these dentin walls. With regards to the coronal seal, MTA was used in the coronal part of the root canal because it is a bioactive material that has an excellent sealing property and considered the golden standard in this type of research ${ }^{(15)}$. DPSCs were used in this study due to their advantages in clinical usage including lower mortality rate, less legal or ethical issues, easy access from extracted teeth, and cryopreservation without losing their multi-differentiation potential (16). Another important feature of dental pulp stem cells are their odontoblastic differentiation potential as they can be induced in vitro to differentiate into cells of odontoblastic phenotype (17). Enzymatic digestion method was used for isolation or as it is widely used in laboratories and preferable to detect, isolate, proliferate and differentiate stem cells (18). Simvastatin (SIM) was used because it is widely prescribed and has a higher bone growth stimulation capability compared with the other hydrophilic Statins. Its long-term systemic administration in humans has been shown to result in increased bone mineral density as well as the anti-inflammatory effect when delivered or applied locally. Methylcellulose is generally regarded as a nontoxic, non-allergic, non-irritating material and is used as a sustained release vehicle for therapeutic drugs ${ }^{(19)}$. Thus, delivery of the drug was controlled via degradation of the hydrogel carrier not by simple diffusion. It is becoming increasingly evident that the ideal scaffold for dental pulp tissue engineering will be injectable, not casted. This is because of the narrow spaces within the root canal and the complexity of its anatomy, particularly in the apical region so hydrogels can penetrate throughout the root canal system ${ }^{(20)}$. Radiographic examination was done periodically over the different evaluation periods to detect tissue changes (radiolucency, apical closure and canal calcifications). While Histologic examination was done to evaluate (inflammatory changes, intracanal tissue changes, hard tissue formation and apical closure).

Regarding inflammatory changes of the tested groups, the results showed initial increasing followed by marked reduction along the different evaluation periods. This indicates the progression of healing of periapical lesion and reduction of inflammatory reaction with time. This mild to moderate inflammation might be attributed to the immediate inflammatory reaction of the periradicular tissues to the treatment protocols. This is in agreement with the findings of inflammatory reaction by wang et al (21) and Pereira MS et al (22) who advocated the presence of mild inflammatory reaction regardless of the new tissue ingrowth following regenerative procedures. Additionally, Inflammation may provide factors to guide the differentiation of stem/progenitor cells in the healing soft tissue into cementoblasts. On the other hand, the reduction of inflammation was an indication of progressed healing of the periapical lesion over time that is in agreement with Gomes-Filho et al.

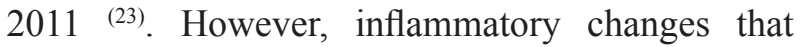
were demonstrated through the combination group (DPSCS+SIM) described as mild inflammations but that in (DPSCs) group showed moderate inflammation. This discrepancy may be attributed to addition of SIM to these groups. This finding was in agreement with Lu D et al (2007) ${ }^{(24)}$ and Miron.VE et al (2007) ${ }^{(25)}$ who revealed that, statins have an anti-inflammatory effect in various tissues and this could help restore the inflamed pulp tissue beside their ability to accelerate reparative dentin formation. 
Regarding intracanal tissue changes of the tested groups, the results showed that DPSCs+SIM group has the highest change followed by DPSCs group. This may be attributed to the potent regenerative power of SIM that allow for synergistic effect when added to DPSCs besides the power of DPSCs as a native progenitor cells that have odontogenic rather than osteogenic development ${ }^{(26)}$. This is in agreement with other study that demonstrated the proliferative ability and developmental potentials of DPSCs beside their ability to develop into distinct tissues representative of the microenvironments from which they were derived in vivo. Also, the potent neurogenicity of dental stem cells may be attributed to their neural crest origin ${ }^{(27)}$. These results were in accordance to Huang et al in $2006{ }^{(28)}$ who stated that when DPSCs seeded onto dentin, some DPSCs convert into odontoblast-like cells with a polarized cell body and a cell process extending into the existing dentinal tubules. The information about the hard tissue produced on dentinal walls and the cells responsible for hard-tissue production is still lacking. An animal study revealed that a cementumlike tissue was deposited on the root canal dentinal walls after regenerative endodontic treatment. This tissue was irregular and was assumed to be responsible for root development ${ }^{(29)}$. The results of this study was in agreement with a study done by Batouli et al. ${ }^{(30)}$ revealed that when DPSCs are seeded onto human dentin surfaces and implanted into immunocompromised mice, reparative dentinlike structure is deposited on the dentin surface. A replacement by regenerative tissues began to appear through a regular arrangement of the pulp tissue architecture. Later on, an improvement of the pulp tissue architecture mimicking the normal pattern was seen after 3 months' evaluation period. These dramatic changes reveal the healing capacity of DPSCs+SIM that might indicate their efficacy and biocompatibility as regenerative materials. These results confirm investigations which reported that DPSCs+SIM causes proliferation of human dental pulp cells and increase the protein expression of osteoprotegerin and alkaline phosphatase activity. Therefore, odontoblast like cells will be produced and pulp-dentin complex will form. Regarding the changes in apical closure of the tested groups, better apical closure recorded for DPSCs+ SIM at 3 months indicating the possible effectiveness of SIM in pulp regeneration along with dentin regeneration.

Regarding radiographic evaluation, Wang et al. (2010) ${ }^{(31)}$ concluded that radiographic findings were not accurate regarding actual increase in root length or thickness. This is with agreement of the results of this study supporting the use of radiographic examination for the confirmation of histologic findings not for accurate measurements. For DPSCs+SIM the highest decrease in periapical radiolucency was recorded at 3 months followed by 1 month. This may be attributed to the effect of simvastatin that promotes osteoblastic differentiation, enhances ALP production and bone mineralization and up-regulates the expression of bone anabolic factors such as VEGF ${ }^{(32)}$. Recent review articles regarding this topic concluded that local delivery of simvastatin (SIM) from biomaterials seems to be more reliable than systemic administration for bone regeneration; however, SIM can either accelerate or retard mineralized new tissue genesis according to the concentration used ${ }^{(33)}$. At low concentrations, these drugs feature pleiotropic effects with mesenchymal stem cells, increasing the expression of several osteo/odontoblastic markers, leading to intense mineralized matrix deposition in vitro ${ }^{(34)}$. Conversely, the higher concentration of SIM may lead to cell death. For DPSCs + Sim canal calcification was significant at 3 months and 1 month when compared with 1 week. The explanation of this finding may also due to the combination of DPSCs+SIM where simvastatin promotes osteoblastic differentiation, enhances ALP production and bone mineralization, and upregulates the expression of bone anabolic factors such as VEGF. While Dental stem cells display multidifferentiation potential, with the capacity to give rise to at least 3 distinct cell lineages: osteo/ 
odontogenic, adipogenic, and neurogenic. Although complete root canal calcification/obliteration is not mentioned as a failure in cases that have undergone regenerative endodontic treatment, it can cause serious challenges in case the involved tooth needs root canal therapy.

\section{CONCLUSIONS}

1. Simvastatin aids in regeneration of infected immature dog's teeth with the ability of producing functional dentin-pulp complex.

2. Dental pulp stem cells have the potential to induce regeneration of infected immature dog's teeth.

3. The outcome of regeneration of immature dog's teeth is evident by apical closure with time.

\section{REFERENCES}

1. Trope M. Regenerative potential of dental pulp. J Endod 2008; 34: S13-7.

2. Petrino J, Boda K, Shambarger S. Challenges in regenerative endodontics: a case series. J Endod 2010; 36:536-41.

3. Garrett I, Gutierrez G, Mundy G. Statins and bone formation. Curr Pharm Des 2001; 7:715-36.

4. Gronthos S, Mankani M, Brahim J, Robey P, Shi S. Postnatal human dental pulp stem cells (DPSCs) in vitro and in vivo. Proc Natl Acad Sci USA 2000; 97:13625-30

5. Huang G. Pulp and dentin tissue engineering and regeneration: current progress. Regen. Med. 2009; 4: 697-707.

6. Shimizu E, Jong G, Partridge N, Rosenberg A., Lin L. Histologic Observation of a Human Immature Permanent Tooth with Irreversible Pulpitis after Revascularization/ Regeneration Procedure. J Endod 2012; 38:1293-1297.

7. Krebsbach P, Kuznetsov S, Satomura K, Emmons R, Rowe D, Robey G. Bone formation in vivo: Comparison of osteogenesis by transplanted mouse and human marrow stromal fibroblasts Transplantation 1997;63: 1059- 1069.

8. Jia W, Zhao W, Yang J, Wang W, Wang X, Ling L. Simvastatin Promotes Dental Pulp Stem Cell-induced Coronal Pulp Regeneration in Pulpotomized Teeth. J Endod 2016; 42:1049-1054.

9. Hargreaves K, Law A. (2011) Regenerative endodontics. In: Hargreaves K, Cohen S, eds. Pathways of the pulp, $10^{\text {th }}$ edn. St.Louis: Mosby Elsevier, pp. 602-19.
10. Cochran P. (2004) Laboratory manual for comparative veterinary anatomy and physiology'. Delmar learning.

11. Hargreaves K, Diogenes A, Teixeira F. Treatment options: biological basis of regenerative endodontic procedures. $\mathrm{J}$ Endod 2013; 39: S30-43.

12. Cvek M, Cleaton-Jones P, Austin J, Lownie J, Kling M, Fatti P. Effect of Topical Application of Doxycycline on Pulp Revascularization and Periodontal Healing in Reimplanted Monkey Incisors. Endod Dent Traumatol. 1990 Aug;6(4):170-6.

13. Roberts-Clark D, Smith A. Angiogenic growth factors in human dentine matrix. Arch Oral Biol 2000; 45:1013-6.

14. Smith A, Murray PE, Sloan A, Matthews J, Zhao S. Transdentinal stimulation of tertiary dentinogenesis. Adv Dent Res 2001; 15:51-4.

15. Torabinejad M, Higa R, McKendry D, Pitt Ford T. Dye leakage of four root end filling materials: effects of blood contamination. J endod 1994; 20:159-63.

16. Gronthos S, Brahim J, Li W. Stem cell properties of human dental pulp stem cells. J Dent Res. 2002; 81:531-535.

17. Couble M, Farges J, Bleicher F, Perrat-Mabillon B, Boudeulle M, Magloire H. Odontoblast differentiation of human dental pulp cells in explant cultures. Calcif Tissue Int 2000; 66:129-138.

18. Chen Y, Zhang v, Fu Q, Liu Y, Wang Z, Nianmin Q. In vitro proliferation and osteogenic differentiation of human dental pulp stem cells in injectable thermo-sensitive chitosan $/ \beta$ glycerophosphate/hydroxyapatite hydrogel. Journal of Biomaterials Applications 2016; 31:317-327.

19. Morris M, Lee Y, Lavin M. Injectable simvastatin in periodontal defects and alveolar ridges: Pilot studies. J Periodontol 2008; 79:1465-1473.

20. Manson J, Dixon D. The influence of solvent processing on polyester bioabsorbable polymers. J Biomater Appl 2012; 26:623-34.

21. Wang Q, Lin X, Lin Z, Liu G, Shan X. Expression of vascular endothelial growth factor in dental pulp of immature and mature permanent teeth in human. Shanghai Kou Qiang Yi Zue. 2007;16(3):285-9.

22. Pereira M, Rossi M, Cardoso C, da Silva J, Bezerra da Silva L, Kuga M, et al. Cellular and molecular tissue response to triple antibiotic intracanal dressing. J Endod. 2014;40(4):499-504.

23. Gomes-Filho J, Costa M, Cintra L. Evaluation of rat alveolar bone response to angelus MTA or experimental light cured mineral trioxide aggregate using fluorochromes. J Endod 2011;37, 250-4. 
24. Lu D, Qu C, Goussev A. Statins increase neurogenesis in the dentate gyrus, reduce delayed neuronal death in the hippocampal CA3 region, and improve spatial learning in rat after traumatic brain injury. J Neurotrauma 2007;24: 1132-46.

25. Miron V, Rajasekharan S, Jarjour A, Zamvil S, Kennedy T, Antel J. Simvastatin regulates oligodendroglial process dynamics and survival. Glia 2007;55: 130-43.

26. Sonoyama W, Liu Y, Fang D, Yamaza T, Seo B, Zhang C. Mesenchymal stem cell-mediated functional tooth regeneration in Swine. PLoS 2006; 1:79.

27. Zhang W, Walboomers X, Shi S, Fan M, Jansen J. Multilineage differentiation potential of stem cells derived from human dental pulp after cryopreservation. Tissue Eng 2006; 12:2813-2823.

28. Huang G, Shagramanova K, Chan S. Formation of odontoblast like cells from cultured human dental pulp cells on dentin in vitro. J Endod 2006b; 32:1066-1073.

29. Wang X., Thibodeau B., Trope M., Lin L., Huang G. Histologic characterization of regenerated tissues in canal space after the revitalization/revascularization procedure of immature dog teeth with apical periodontitis. J Endod $2010 ; 36: 56-63$

30. Batouli S., Miura M., Brahim J., Tsutsui T., Fisher L., Gronthos S, Robey P., Shi S. Comparison of stem cellmediated osteogenesis and dentinogenesis. J Dent Res 2003;82:976-981.

31. Wang X., Thibodeau B., Trope M. Histologic characterization of regenerated tissues in canal space after the revitalization/revascularization procedure of immature dog teeth with apical periodontitis. J Endod 2010; 36:56-63.

32. Kok S., Lee Y. Simvastatin as a novel strategy to alleviate periapical lesions. J Endod 2009; 35:657-62.

33. Kheirallah M., Almeshaly A. Simvastatin, dosage, and delivery system for supporting bone regeneration, an update review. J Oral Maxillofac Surg Med Pathol 2016;28: 206-9.

34. Zijah V., Salehi R., Aghazadeh M. Towards optimization of odonto/osteogenic bioengineering:in vitro comparison of simvastatin, sodium fluoride, melanocytestimulating hormone. In Vitro Cell Dev Biol Anim 2017; 53:502-12. 